\title{
Synthesis and Antifungal Activity of New Nitrobenzofuran Derivatives
}

\author{
Ahmet Çağrı KARABURUN ${ }^{1 *}$
}

\begin{abstract}
The object of present study is to assess anticandidal activities of some new aryl (5nitrobenzofuran-2-yl)keton and ketoxime compounds. Eight aryl (5-nitrobenzofuran-2-yl)ketones and ketoximes were synthesized. IR, ${ }^{1} \mathrm{H}-\mathrm{NMR}$ and HR-MS spectroscopic data, performed the structure elucidation of the synthesized compounds. Anticandidal activities of the all synthesized compounds were studied. Compound 2c bearing methoxy group on phenyl ring showed the highest activity with MIC value of $3.12 \mu \mathrm{g} \mathrm{mL}^{-1}$ against Candida albicans and Candida glabrata. None of the compounds activity results were equal to or better than that of the control compounds ketoconazole and fluconazole. However, compound 2c displayed a promising anticandidal activity.
\end{abstract}

Keywords: Aryl (5-nitrobenzofuran-2-yl)ketones, aryl (5-nitrobenzofuran-2-yl)ketoximes, anticandidal activity.

\section{Yeni Nitrobenzofuran Türevlerinin Sentezi ve Antifungal Etkileri}

ÖZET: Bu çalışmada bazı yeni aril (5-nitrobenzofuran-2-il)keton ve ketoksim bileşiklerinin antikandidal etkinliklerinin değerlendirilmesi amaçlanmıştır. Sekiz aril (5-nitrobenzofuran-2-il)keton ve ketoksimi sentezlenmiştir. Sentezlenen bileşiklerin yapı aydınlatmaları IR, ${ }^{1} \mathrm{H}-\mathrm{NMR}$ ve HR-MS spektroskopik verileri ile gerçekleştirilmiştir. Bileşiklerin antikandidal etkinlikleri incelenmiştir. Fenil halkası üzerinde metoksi grubu taşıyan bileşik 2c, $3.12 \mu \mathrm{g} \mathrm{mL}^{-1}$ Mİ değeri ile Candida albicans ve Candida glabrata'ya karşı en yüksek aktiviteyi göstermiştir. Hiçbir bileşik kontrol bileşikleri olan ketokonazol ve flukonazole eş ya da daha iyi aktivite sergileyememiştir. Ancak, bileşik 2c'nin aktivite sonuçları umut verici olarak değerlendirilmektedir.

Anahtar Kelimeler: Aril (5-nitrobenzofuran-2-il)ketonlar, aril (5-nitrobenzofuran-2-il)ketoksimler, antikandidal aktivite.

\footnotetext{
${ }^{1}$ Ahmet Çağrı KARABURUN (Orcid ID: 0000-0002-2503-3824), Anadolu University, Faculty of Pharmacy, Department of Pharmaceutical Chemistry, Eskişehir, Türkiye

Sorumlu yazar/Corresponding Author: Ahmet Çağrı KARABURUN, e-mail: ackarabu@anadolu.edu.tr
} 


\section{INTRODUCTION}

Worldwide, the physicians draw attention on fungal infections due to the frequency of suffered patients from fungal diseases (Valerio et al., 2015; Benedict et al., 2017). It has been reported by clinical data that the incidence of fungal infections are increased in patients with cancer and tuberculosis since immunity falls or disappears (Sipsas and Kontoyiannis, 2012; Osman et al., 2013). Also, the development of multi-drug and pandrug resistances cause insufficient treatment, especially for one of the largest group of fungal pathogens, Candida species which are also the most reported species regarding resistance development (Dos Santos Abrantes et al., 2014; Dimopoulos et al., 2015; Healey et al., 2016). Thus, researchers have focused on the development of more effective agents against opportunistic fungi and they tried to inhibit the development of resistance. On the other hand, it is petitive that these antimicrobial agents to be developed have broad spectrum with low cytotoxicity against human cells (Miyazaki et al., 2011; Ravu et al., 2013).

In medicinal chemistry, benzofuran ring system has a special importance because of its pharmacological properties such as analgesic (Rádl et al., 2000), antinociceptive (Goncalves et al., 2012), antibacterial (Jiang et al., 2011), antifungal (Demirayak et al., 2016), antiviral (Engler et al., 1996), antihypertensive (de Souza Nunes et al., 2014) and anticancer (Mao et al., 2016) activities. Its antifungal activity is supported by recent studies (Telvekar et al., 2012; Geronikaki et al., 2013), which reported the clinical uses of griseofulvin (Grover et al., 2012), in which benzofuran is located as a core structure. Furthermore, griseofulvin has been indicated successful on patients in comparison with other antifungal agents independent of the fungus type (Wallace, 1977; Shemer et al., 2013).
Oximes are used as a constitutive structure to gain biologically active compounds and they are a preference for bioisosteric replacement and protection of carbonyl groups (Primožič et al., 2014). Moreover, having the easy modification for substitutions on oxygen atom, it could increase or maintain the antifungal activity vis-àvis carbonyl group (Kirilmis et al., 2008).

Thus, for the above mentioned reasons eight compounds (1a-1d, 2a-2d), involved 5nitrobenzofuran as core structure, were synthesized and analyzed for antifungal activity.

\section{MATERIALS AND METHODS}

\section{Chemistry}

All chemicals used in the syntheses were purchased either from Sigma-Aldrich Chemicals (Sigma-Aldrich Corp., St. Louis, MO, USA) or Merck Chemicals (Merck KGaA, Darmstadt, Germany). Melting points were determined by using an Electrothermal 9100 digital melting point apparatus and were presented as uncorrected. ${ }^{1} \mathrm{H}-\mathrm{NMR}$ spectra was recorded by a Bruker $300 \mathrm{MHz}$ digital FT-NMR spectrometer (Bruker Bioscience, Billerica, MA, USA) in DMSO- $d_{6}$. In the NMR spectra, splitting patterns were designated as follows: s: singlet; $\mathrm{d}$ : doublet; t: triplet; $\mathrm{m}$ : multiplet. Coupling constants $(J)$ were reported as Hertz. The IR spectra of the compounds were recorded using an IRAffinity-1S Fourier transform IR (FTIR) spectrometer (Shimadzu, Kyoto, Japan). Mass spectra were recorded on an LCMS-IT-TOF (Shimadzu, Kyoto, Japan) by using ESI method. The purities of compounds were checked by TLC on silica gel 60 F254 (Merck KGaA, Darmstadt, Germany). Aryl (5-nitrobenzofuran2-yl)ketone derivatives were synthesized in accordance with previous methods (Pestellini et al., 1988). 
New compounds were obtained with the reactions depicted in Scheme 1.<smiles>O=Cc1cc([N+](=O)[O-])ccc1O</smiles><smiles>[R]c1ccc(C(=O)CBr)cc1</smiles><smiles>[R]c1ccc(C(=O)c2cc3cc([N+](=O)[O-])ccc3o2)cc1</smiles>

1a-d $+\mathrm{H}_{2} \mathrm{NOH} \cdot \mathrm{HCl}$<smiles>[R]c1ccc(/C(=N\O)c2cc3cc([N+](=O)[O-])ccc3o2)cc1</smiles>

R: $\mathrm{H}, \mathrm{CH}_{3}, \mathrm{OCH}_{3}, \mathrm{Cl}$ a: $\mathrm{K}_{2} \mathrm{CO}_{3} / \mathrm{CH}_{3} \mathrm{CN} /$ reflux b: $\mathrm{CH}_{3} \mathrm{COONa} / \mathrm{C}_{2} \mathrm{H}_{5} \mathrm{OH} /$ reflux

Scheme 1. Synthetic procedure for the compounds

Some characteristics of the synthesized compounds were given in Table $\mathbf{1 .}$

Table 1. Some characteristics of the synthesized compounds

\begin{tabular}{lccccc}
\hline Compounds & $\mathbf{R}$ & m.p. $\left({ }^{\circ} \mathbf{C}\right)$ & Yield (\%) & Formulae & Mol. Weight \\
\hline $\mathbf{1 a}$ & $\mathrm{H}$ & $205-6^{*}$ & 78 & $\mathrm{C}_{15} \mathrm{H}_{9} \mathrm{NO}_{4}$ & 267.24 \\
$\mathbf{1 b}$ & $\mathrm{CH}_{3}$ & $214-5$ & 82 & $\mathrm{C}_{16} \mathrm{H}_{11} \mathrm{NO}_{4}$ & 281.27 \\
$\mathbf{1 c}$ & $\mathrm{OCH}_{3}$ & $233-4$ & 75 & $\mathrm{C}_{16} \mathrm{H}_{11} \mathrm{NO}_{5}$ & 297.27 \\
$\mathbf{1 d}$ & $\mathrm{Cl}$ & $209-10$ & 87 & $\mathrm{C}_{15} \mathrm{H}_{8} \mathrm{ClNO}_{4}$ & 301.69 \\
$\mathbf{2 a}$ & $\mathrm{H}$ & $126-7$ & 75 & $\mathrm{C}_{15} \mathrm{H}_{10} \mathrm{~N}_{2} \mathrm{O}_{4}$ & 282.26 \\
$\mathbf{2 b}$ & $\mathrm{CH}_{3}$ & $240-1$ & 80 & $\mathrm{C}_{16} \mathrm{H}_{12} \mathrm{~N}_{2} \mathrm{O}_{4}$ & 296.29 \\
$\mathbf{2 c}$ & $\mathrm{OCH}_{3}$ & $263-4$ & 72 & $\mathrm{C}_{16} \mathrm{H}_{12} \mathrm{~N}_{2} \mathrm{O}_{5}$ & 312.28 \\
$\mathbf{2 d}$ & $\mathrm{Cl}$ & $234-5$ & 82 & $\mathrm{C}_{15} \mathrm{H}_{9} \mathrm{ClN}_{2} \mathrm{O}_{4}$ & 316.70 \\
\hline
\end{tabular}

Lit (Vinh et al., 1999) m.p. $203{ }^{\circ} \mathrm{C}$.

Synthesis of Aryl (5-nitrobenzofuran-2yl)ketones (1a-d)

Aryl (5-nitrobenzofuran-2-yl)ketone derivatives were synthesized in accordance with previous methods (Pestellini et al., 1988).

\section{(5-Nitrobenzofuran-2-yl)(phenyl)methanone} (1a)

Yield: $78 \%$, M.P. $=205-6^{\circ} \mathrm{C}$, FTIR (ATR, $\left.\mathrm{cm}^{-1}\right)$ : 3096, $1645(\mathrm{C}=\mathrm{O}), 1090,800,696 .{ }^{1} \mathrm{H}-$ NMR (300 MHz, Acetone-d 6 ): $\delta=7.63-7.68$ $(2 \mathrm{H}, \mathrm{m}$, phenyl $\mathrm{CH}), 7.75-7.77(1 \mathrm{H}, \mathrm{m}$, phenyl CH), 7.96 (1H, s, BF-H), 7.98 (1H, d, J=9.36 Hz,
BF-H), 8.11- 8.14 (2H, m, phenyl CH), 8.48 $(1 \mathrm{H}, \mathrm{dd}, \mathrm{J}=9.12 \mathrm{~Hz}-2.43 \mathrm{~Hz}, \mathrm{BF}-\mathrm{H}), 8.85(1 \mathrm{H}, \mathrm{d}$, $\mathrm{J}=2.37 \mathrm{~Hz}, \mathrm{BF}-\mathrm{H})$. HRMS (m/z): $[\mathrm{M}+\mathrm{H}]^{+}$calcd for $\mathrm{C}_{15} \mathrm{H}_{9} \mathrm{NO}_{4}$ : 268.0604; found 268.0614.

\section{(5-Nitrobenzofuran-2-yl)(p-tolyl)methanone} (1b)

Yield: $82 \%$, M.P. $=214-5{ }^{\circ} \mathrm{C}$, FTIR (ATR, $\left.\mathrm{cm}^{-1}\right)$ : 2986, $1645(\mathrm{C}=\mathrm{O}), 1051,833,746 .{ }^{1} \mathrm{H}-$ NMR $\left(300 \mathrm{MHz}\right.$, Acetone- $\left.\mathrm{d}_{6}\right): \delta=2.49(3 \mathrm{H}, \mathrm{s}$, $\left.\mathrm{CH}_{3}\right), 7.47(2 \mathrm{H}, \mathrm{d}, \mathrm{J}=8.19 \mathrm{~Hz}$, methylphenyl $\mathrm{CH}), 7.93(1 \mathrm{H}, \mathrm{s}, \mathrm{BF}-\mathrm{H}), 7.97(1 \mathrm{H}, \mathrm{d}, \mathrm{J}=9.15$ $\mathrm{Hz}, \quad \mathrm{BF}-\mathrm{H}), \quad 8.05 \quad(2 \mathrm{H}, \quad \mathrm{d}, \quad \mathrm{J}=8.19 \mathrm{~Hz}$, 
methylphenyl CH), $8.48(1 \mathrm{H}, \mathrm{dd}, \mathrm{J}=9.15 \mathrm{~Hz}-$ $2.40 \mathrm{~Hz}$ BF-H), 8.84 (1H, d, J=2.37 Hz, BF-H). HRMS $(\mathrm{m} / \mathrm{z}):[\mathrm{M}+\mathrm{H}]^{+}$calcd for $\mathrm{C}_{16} \mathrm{H}_{11} \mathrm{NO}_{4}$ : 282.0761; found 282.0770.

\section{(4-Methoxyphenyl)(5-nitrobenzofuran-2- yl)methanone (1c)}

Yield: $75 \%$, M.P. $=233-4{ }^{\circ} \mathrm{C}$, FTIR (ATR, $\left.\mathrm{cm}^{-1}\right)$ : 2936, $1639(\mathrm{C}=\mathrm{O}), 1063,800,752 .{ }^{1} \mathrm{H}-$ NMR $\left(300 \mathrm{MHz}\right.$, Acetone-d $\left.\mathrm{d}_{6}\right): \delta=3.97(3 \mathrm{H}, \mathrm{s}$, $\left.\mathrm{OCH}_{3}\right), 7.17(2 \mathrm{H}, \mathrm{d}, \mathrm{J}=8.94 \mathrm{~Hz}$, methoxyphenyl $\mathrm{CH}), 7.92(1 \mathrm{H}, \mathrm{s}, \mathrm{BF}-\mathrm{H}), 7.97(1 \mathrm{H}, \mathrm{d}, \mathrm{J}=9.09$ $\mathrm{Hz}, \quad \mathrm{BF}-\mathrm{H}), \quad 8.18 \quad(2 \mathrm{H}, \quad \mathrm{d}, \quad \mathrm{J}=8.94 \quad \mathrm{~Hz}$, methoxyphenyl $\mathrm{CH}), 8.47(1 \mathrm{H}$, dd, J=9.15 Hz$2.40 \mathrm{~Hz}, \mathrm{BF}-\mathrm{H}), 8.85$ (1H, d, J=2.34 Hz, BF-H). HRMS $(\mathrm{m} / \mathrm{z}):[\mathrm{M}+\mathrm{H}]^{+}$calcd for $\mathrm{C}_{16} \mathrm{H}_{11} \mathrm{NO}_{5}$ : 298.0710; found 298.0723.

\section{(4-Chlorophenyl)(5-nitrobenzofuran-2- yl)methanone (1d)}

Yield: $87 \%$, M.P. $=209-10{ }^{\circ} \mathrm{C}$, FTIR $\left(\mathrm{ATR}, \mathrm{cm}^{-1}\right)$ : 3093, $1661(\mathrm{C}=\mathrm{O}), 1092,800,748$. ${ }^{1} \mathrm{H}-\mathrm{NMR}\left(300 \mathrm{MHz}\right.$, Acetone- $\left.\mathrm{d}_{6}\right): \delta=7.70(2 \mathrm{H}$, d, J=8.55 Hz, chlorophenyl $\mathrm{CH}), 7.97-8.00(2 \mathrm{H}$, m, BF-H), 8.17 (2H, d, J=8.55 Hz, chlorophenyl CH), 8.49 (1H, dd, J=9.00 Hz-2.22 Hz, BF-H), $8.85(1 \mathrm{H}, \mathrm{d}, \mathrm{J}=2.40 \mathrm{~Hz}, \mathrm{BF}-\mathrm{H}) . \operatorname{HRMS}(\mathrm{m} / \mathrm{z})$ : $[\mathrm{M}+\mathrm{H}]^{+}$calcd for $\mathrm{C}_{15} \mathrm{H}_{8} \mathrm{NO}_{4} \mathrm{Cl}$ : 302.0215; found 302.0225 .

\section{Synthesis of Aryl (5-nitrobenzofuran-2- yl)ketoximes (2a-d)}

The suitable aryl (5-nitrobenzofuran-2-yl) ketone derivative $(5 \mathrm{mmol})$, 1a-d, was heated in ethanol with hydroxylamine hydrochloride (7 mmol) for 3 hours with catalyze of anhydrous sodium acetate $(7 \mathrm{mmol})$. After completion of reaction, the mixture was allowed to cool for crystallization. The precipitated product was filtered and recrystallized from ethanol.

\section{(E/Z)-(5-nitrobenzofuran-2- \\ $y l)($ phenyl)methanone oxime (2a)}

Yield: $75 \%$, M.P. $=126-7^{\circ} \mathrm{C}$, FTIR (ATR, $\left.\mathrm{cm}^{-1}\right): 3379(\mathrm{O}-\mathrm{H}), 2988,1634(\mathrm{C}=\mathrm{O}), 1013$,
820, 696. ${ }^{1} \mathrm{H}-\mathrm{NMR}\left(300 \mathrm{MHz}\right.$, Acetone-d $\left.\mathrm{d}_{6}\right): \delta=$ 7.47- $7.65(5 \mathrm{H}, \mathrm{m}$, phenyl $\mathrm{CH}), 7.75(1 \mathrm{H}, \mathrm{d}$, $\mathrm{J}=9.32 \mathrm{~Hz}, \mathrm{BF}-\mathrm{H}), 8.08(1 \mathrm{H}, \mathrm{s}, \mathrm{BF}-\mathrm{H}), 8.33(1 \mathrm{H}$, dd, J=9.12 Hz-2.40 Hz, BF-H), $8.77(1 \mathrm{H}, \mathrm{d}, \mathrm{J}=$ $2.37 \mathrm{~Hz}, \mathrm{BF}-\mathrm{H})$. HRMS (m/z): $[\mathrm{M}+\mathrm{H}]^{+}$calcd for $\mathrm{C}_{15} \mathrm{H}_{10} \mathrm{~N}_{2} \mathrm{O}_{4}$ : 283.0713; found 283.0719.

\section{(E/Z)-(5-nitrobenzofuran-2-yl)(p-} tolyl)methanone oxime (2b)

Yield: $80 \%$, M.P. $=240-1{ }^{\circ} \mathrm{C}$, FTIR (ATR, $\left.\mathrm{cm}^{-1}\right)$ : $3202(\mathrm{O}-\mathrm{H}), 3001,1622(\mathrm{C}=\mathrm{O}), 1013$, 818, 685. ${ }^{1} \mathrm{H}-\mathrm{NMR}\left(300 \mathrm{MHz}\right.$, Acetone- $\left.\mathrm{d}_{6}\right): \delta=$ $2.40\left(3 \mathrm{H}, \mathrm{s}, \mathrm{CH}_{3}\right), 7.29(2 \mathrm{H}, \mathrm{d}, \mathrm{J}=8.01 \mathrm{~Hz}$, methylphenyl $\mathrm{CH}), 7.52(2 \mathrm{H}, \mathrm{d}, \mathrm{J}=8.13 \mathrm{~Hz}$, methylphenyl CH), $7.77(1 \mathrm{H}, \mathrm{d}, \mathrm{J}=8.79 \mathrm{~Hz}, \mathrm{BF}-$ H), $8.05(1 \mathrm{H}, \mathrm{s}, \mathrm{BF}-\mathrm{H}), 8.32(1 \mathrm{H}, \mathrm{dd}, \mathrm{J}=8.79 \mathrm{~Hz}-$ $2.40 \mathrm{~Hz}, \mathrm{BF}-\mathrm{H}), 8.76(1 \mathrm{H}, \mathrm{d}, \mathrm{J}=2.37 \mathrm{~Hz}, \mathrm{BF}-\mathrm{H})$. HRMS (m/z): $[\mathrm{M}+\mathrm{H}]^{+}$calcd for $\mathrm{C}_{16} \mathrm{H}_{12} \mathrm{~N}_{2} \mathrm{O}_{4}$ : 297.0870; found 297.0877.

(E/Z)-(4-methoxyphenyl)(5-nitrobenzofuran-2$y$ l)methanone oxime (2c)

Yield: $72 \%$, M.P. $=263-4{ }^{\circ} \mathrm{C}$, FTIR (ATR, $\left.\mathrm{cm}^{-1}\right): 3227(\mathrm{O}-\mathrm{H}), 2997,1639(\mathrm{C}=\mathrm{O}), 1018$, 810, 750. ${ }^{1} \mathrm{H}-\mathrm{NMR}\left(300 \mathrm{MHz}\right.$, Acetone- $\left.\mathrm{d}_{6}\right): \delta=$ $3.88\left(3 \mathrm{H}, \mathrm{s}, \mathrm{OCH}_{3}\right), 7.03(2 \mathrm{H}, \mathrm{d}, \mathrm{J}=8.94 \mathrm{~Hz}$, methoxyphenyl $\mathrm{CH}), 7.58(2 \mathrm{H}, \mathrm{d}, \mathrm{J}=8.94 \mathrm{~Hz}$, methoxyphenyl $\mathrm{CH}), 7.78(1 \mathrm{H}, \mathrm{d}, \mathrm{J}=9.09 \mathrm{~Hz}$, BF-H), 8.03 (1H, s, BF-H), 8.33 (1H, dd, J=9.09 Hz-2.40 Hz, BF-H), $8.77(1 \mathrm{H}, \mathrm{d}, \mathrm{J}=2.34 \mathrm{~Hz}$, BF-H). HRMS (m/z): $[\mathrm{M}+\mathrm{H}]^{+}$calcd for $\mathrm{C}_{16} \mathrm{H}_{12} \mathrm{~N}_{2} \mathrm{O}_{5}$ : 313.0819; found 313.0830.

\section{(E/Z)-(4-chlorophenyl)(5-nitrobenzofuran-2-} $y l) m e t h a n o n e ~ o x i m e ~(2 d)$

Yield: $82 \%$, M.P. $=234-5{ }^{\circ}$ C, FTIR (ATR, $\left.\mathrm{cm}^{-1}\right): 3177(\mathrm{O}-\mathrm{H}), 3001,1651(\mathrm{C}=\mathrm{O}), 1022$, 818, 685. ${ }^{1} \mathrm{H}-\mathrm{NMR}\left(300 \mathrm{MHz}\right.$, Acetone- $\left.\mathrm{d}_{6}\right): \delta=$ $7.53(2 \mathrm{H}, \mathrm{d}, \mathrm{J}=8.61 \mathrm{~Hz}$, chlorophenyl $\mathrm{CH}), 7.69$ $(2 \mathrm{H}, \mathrm{d}, \mathrm{J}=8.61 \mathrm{~Hz}$, chlorophenyl $\mathrm{CH}), 7.79(1 \mathrm{H}$, d, J=9.06 Hz, BF-H), $8.11(1 \mathrm{H}, \mathrm{s}, \mathrm{BF}-\mathrm{H}), 8.33$ $(1 \mathrm{H}, \mathrm{dd}, \mathrm{J}=9.09 \mathrm{~Hz}-2.40 \mathrm{~Hz}, \mathrm{BF}-\mathrm{H}), 8.78(1 \mathrm{H}, \mathrm{d}$, $\mathrm{J}=2.37 \mathrm{~Hz}, \mathrm{BF}-\mathrm{H}) . \mathrm{HRMS}(\mathrm{m} / \mathrm{z}):[\mathrm{M}+\mathrm{H}]^{+}$calcd for $\mathrm{C}_{15} \mathrm{H}_{9} \mathrm{~N}_{2} \mathrm{O}_{4} \mathrm{Cl}$ : 317.0324; found 317.0333. 


\section{Antifungal Activity}

Anticandidal activity, according to the protocol of the EUCAST (Rodriguez-Tudela et al., 2008), was performed for gained final compounds 1a-d, 2a-d against Candida albicans (ATCC 24433), Candida krusei (ATCC 6258), Candida parapsilosis (ATCC 22019) and Candida glabrata (ATCC 90030). Synthesized compounds as well as ketoconazole and fluconazole as reference drugs were tested. The minimum inhibitory concentrations (MICs) of the all synthesized compounds were determined by fluorometric measurements (Palomino et al., 2002; Borra et al., 2009). Table 2 presents MIC values of all compounds as antifungal results.

Table 2. $\mathrm{MIC}_{50}\left(\mu \mathrm{g} \mathrm{mL}^{-1}\right)$ values of compounds (1a-d, 2a-d)

\begin{tabular}{ccccc}
\hline Compounds & Candida albicans & Candida glabrata & Candida krusei & Candida parapsilosis \\
\hline 1a & 50 & 50 & 100 & 100 \\
$\mathbf{1 b}$ & 25 & 25 & 50 & 25 \\
$\mathbf{1 c}$ & 12.50 & 25 & 50 & 50 \\
$\mathbf{1 d}$ & 12.50 & 50 & 100 & 100 \\
$\mathbf{2 a}$ & 25 & 50 & 25 & 100 \\
$\mathbf{2 b}$ & 12.50 & 12.50 & 25 & 50 \\
$\mathbf{2 c}$ & 3.12 & 3.12 & 6.25 & 12.50 \\
$\mathbf{2 d}$ & 6.25 & 12.50 & 25 & 12.50 \\
\hline Ketoconazole & 0.78 & 1.56 & 1.56 & 1.56 \\
\hline Fluconazole & 0.78 & 1.56 & 1.56 & 0.78 \\
\hline
\end{tabular}

\section{RESULTS AND DISCUSSION}

\section{Chemistry}

The reactions, depicted in scheme were followed for the synthesis of aryl (5nitrobenzofuran-2-yl)ketoxime compounds. A slightly altered Rap-Störmer Reaction gave the ketonic compounds, 1a-d, (Pestellini et al., 1988). The ketones were allowed to react with hydroxylamine hydrochloride to obtain the oxime derivatives (2a-d). In evaluation of the spectral data of the synthesized compounds, all data were obtained as expected. In IR spectra; ketone compounds showed characteristic carbonyl stretching bands at about 1640-1660 $\mathrm{cm}^{-1}$ region, also oxime compounds showed characteristic O-H bands at about $3200-3400 \mathrm{~cm}^{-}$

${ }^{1}$ region. Characteristic nitro group symmetric and asymmetric stretching bands were observed for all compounds. In ${ }^{1} \mathrm{H}-\mathrm{NMR}$ spectra; all aliphatic and aromatic protons were observed as expected with corresponding integral values.
However, oxime hydrogens which were effortlessly identified in IR spectra, could not be observed in NMR. It may be attributed to the proton exchange with deutero solvent used in NMR. It is also of consideration that the electron withdrawing effect of nitro group increases the acidity of the oxime which facilitates the proton exchange of this group. For all compounds HRMS spectra showed exact molecular mass and formula.

\section{Antifungal Activity}

According to antifungal activity results, all compounds displayed anticandidal activity. Compounds with oxime moiety (2a-2d) were found more active than their carbonyl substitution derivatives (1a-1d) against Candida species. In fact, compound $\mathbf{2 c}$ was found to be the most potent derivative in the series against all Candida species with $\mathrm{MIC}_{50}$ values of 3.12 and $12.50 \mu \mathrm{g} \mathrm{mL}^{-1}$. Moreover, it can be seen that 
compound 2d showed remarkable activity against Candida albicans with a $\mathrm{MIC}_{50}$ value of $6.25 \mu \mathrm{g} \mathrm{mL}^{-1}$. Also, any substitution at the 4th position of the benzene ring increased anticandidal activity, particularly 4-methoxy substitution was found two times more active than other substitutions. On the other hand, none of the synthesized compounds showed higher activity than the standard drug.

In consideration of the antifungal activity results, it is seen that ketone compounds showed very little activity on tested fungi. When the carbonyl group converted to its oxime, the activity elevated for all compounds. In addition, effect of the substituent positioned on the phenyl ring is crucial for activity. Especially compound 2c displayed a promising anticandidal activity. Methoxy substitution on the phenyl ring resulted in the highest activity among the synthesized compounds.

\section{CONCLUSION}

None of the compounds activity results was equal to or better than that of the control compounds ketoconazole and fluconazole. However, compound $\mathbf{2 c}$ displayed a promising anticandidal activity. In general, it is expected that oxime derivatives of nitrobenzofuran might be promising antifungal candidates. Hence, with further study and synthesis of new oxime derivatives, better nitrobenzofuran antifungal agents can possibly be obtained. This in turn can contribute to the inhibition of the ongoing fungal resistance development to currently available antifungal agents.

\section{ACKNOWLEDGMENTS}

Financial contribution of Anadolu University Scientific Projects Fund (Project No.: 1807S253) is gratefully acknowledged.

\section{REFERENCES}

Benedict K, Richardson M, Vallabhaneni S, Jackson BR, Chiller T, 2017. Emerging issues, challenges, and changing epidemiology of fungal disease outbreaks. The Lancet Infectious Diseases, 17 (12): e403-e11.

Borra RC, Lotufo MA, Gagioti SM, Barros Fde M, Andrade PM, 2009. A simple method to measure cell viability in proliferation and cytotoxicity assays. Brazilian Oral Research, 23 (3): 255-262.

de Souza Nunes JP, da Silva KA, da Silva GF, Quintao NL, Correa R, Cechinel-Filho V, et al, 2014. The antihypersensitive and antiinflammatory activities of a benzofuranone derivative in different experimental models in mice: the importance of the protein kinase $\mathrm{C}$ pathway. Anesthesia \& Analgesia, 119 (4): 836-846.

Demirayak S, Yurttas L, Karaburun AC, GundogduKaraburun N, Kayagil I, 2016. Synthesis and antiproliferative activity of 2-arylidene 6-(2aryl-2-oxoethoxy)benzofuran-3-one

derivatives. Letters in Drug Design \& Discovery, 13 (6): 563-569.

Dimopoulos G, Koulenti D, Tabah A, Poulakou G, Vesin A, Arvaniti K, et al, 2015. Bloodstream infections in ICU with increased resistance: epidemiology and outcomes. Minerva Anestesiologica, 81 (4): 405-418.

Dos Santos Abrantes PM, McArthur CP, Africa CW, 2014. Multi-drug resistant oral Candida species isolated from HIV-positive patients in South Africa and Cameroon. Diagnostic Microbiology and Infectious Disease, 79 (2): 222-227.

Engler TA, LaTessa KO, Iyengar R, Chai W, Agrios K, 1996. Stereoselective syntheses of substituted pterocarpans with anti-HIV activity, and 5-aza-/5-thia-pterocarpan and 2aryl-2,3-dihydrobenzofuran analogues. Bioorganic \& Medicinal Chemistry, 4 (10): 1755-1769. 
Geronikaki A, Fesatidou M, Kartsev V, Macaev F, 2013. Synthesis and biological evaluation of potent antifungal agents. Current Topics in Medicinal Chemistry, 13 (21): 2684-2733.

Goncalves CJ, Lenoir AS, Padaratz P, Correa R, Niero R, Cechinel-Filho V, et al, 2012. Benzofuranones as potential antinociceptive agents: structure-activity relationships. European Journal of Medicinal Chemistry, 56: 120-126.

Grover C, Arora P, Manchanda V, 2012. Comparative evaluation of griseofulvin, terbinafine and fluconazole in the treatment of tinea capitis. International Journal of Dermatology, 51 (4): 455-458.

Healey KR, Zhao Y, Perez WB, Lockhart SR, Sobel JD, Farmakiotis D, et al, 2016. Prevalent mutator genotype identified in fungal pathogen Candida glabrata promotes multi-drug resistance. Nature Communications, 7: 11128.

Jiang X, Liu W, Zhang W, Jiang F, Gao Z, Zhuang $\mathrm{H}$, et al, 2011. Synthesis and antimicrobial evaluation of new benzofuran derivatives. European Journal of Medicinal Chemistry, 46 (8): 3526-3530.

Kirilmis C, Ahmedzade M, Servi S, Koca M, Kizirgil A, Kazaz C, 2008. Synthesis and antimicrobial activity of some novel derivatives of benzofuran: Part 2. The synthesis and antimicrobial activity of some novel 1-(1benzofuran-2-yl)-2-mesitylethanone derivatives. European Journal of Medicinal Chemistry, 43 (2): 300-308.

Mao ZW, Zheng X, Lin YP, Hu CY, Wang XL, Wan $\mathrm{CP}$, et al, 2016. Design, synthesis and anticancer activity of novel hybrid compounds between benzofuran and $N$-aryl piperazine. Bioorganic \& Medicinal Chemistry Letters, 26 (15): 3421-3424.
Miyazaki M, Horii $\mathrm{T}$, Hata $\mathrm{K}$, Watanabe NA, Nakamoto K, Tanaka K, et al, 2011. In vitro activity of E1210, a novel antifungal, against clinically important yeasts and molds. Antimicrobial Agents and Chemotherapy, 55 (10): 4652-4658.

Osman NM, Gomaa AA, Sayed NM, Abd el aziz AA, 2013. Microarray detection of fungal infection in pulmonary tuberculosis. Egyptian Journal of Chest Diseases and Tuberculosis, 62 (1): 151-157.

Palomino JC, Martin A, Camacho M, Guerra H, Swings J, Portaels F, 2002. Resazurin microtiter assay plate: simple and inexpensive method for detection of drug resistance in Mycobacterium tuberculosis. Antimicrobial Agents and Chemotherapy, 46 (8): 2720-2722.

Pestellini V, Giolitti A, Pasqui F, Abelli L, Cutrufo C, De Salvia G, et al, 1988. Synthesis and hypolipidemic activity of new substituted (benzofuran-2-yl)-phenyl-carbinols. European Journal of Medicinal Chemistry, 23 (2): 203206.

Primožič I, Hrenar T, Baumann K, Krišto L, Križić I, Tomić S, 2014. Mechanochemical and conformational study of $N$-heterocyclic carbonyl-oxime transformations. Croatica Chemica Acta, 87 (2):153-160.

Rádl S, Hezký P, Konvička P, Krejčí I, 2000. Synthesis and analgesic activity of some substituted 1-benzofurans and 1benzothiophenes. Collection of Czechoslovak Chemical Communications, 65 (7): 10931108 .

Ravu RR, Chen YL, Jacob MR, Pan X, Agarwal AK, Khan SI, et al, 2013. Synthesis and antifungal activities of miltefosine analogs. Bioorganic \& Medicinal Chemistry Letters, 23 (17): 48284831. 
Rodriguez-Tudela JL, Arendrup MC, Barchiesi F, Bille J, Chryssanthou E, Cuenca-Estrella M, Dannaoui E, Denning DW, Donnelly JP, Dromer F, Fegeler W, 2008. EUCAST definitive document EDef 7.1: method for the determination of broth dilution MICs of antifungal agents for fermentative yeasts. Clinical Microbiology and Infection, 14 (4): 398-405.

Shemer A, Plotnik IB, Davidovici B, Grunwald MH, Magun R, Amichai B, 2013. Treatment of tinea capitis - griseofulvin versus fluconazole a comparative study. Journal der Deutschen Dermatologischen Gesellschaft, 11 (8): 737741 .

Sipsas NV, Kontoyiannis DP, 2012. Invasive fungal infections in patients with cancer in the Intensive Care Unit. International Journal of Antimicrobial Agents, 39 (6): 464-471.

Telvekar VN, Belubbi A, Bairwa VK, Satardekar K, 2012. Novel $N$ '-benzylidene benzofuran-3carbohydrazide derivatives as antitubercular and antifungal agents. Bioorganic \& Medicinal Chemistry Letters, 22 (6): 2343-2346.
Valerio M, Vena A, Bouza E, Reiter N, Viale P, Hochreiter M, et al, 2015. How much European prescribing physicians know about invasive fungal infections management? BMC Infectious Diseases, 15: 80.

Vinh TK, Ahmadi M, Lopez Delgado PO, Fernandez Perez S, Walters HM, Smith HJ, Nicholls PJ, Simons C, 1999. 1-[(Benzofuran-2-yl) phenylmethyl]-triazoles and -tetrazoles potent competitive inhibitors of aromatase. Bioorganic \& Medicinal Chemistry Letters, 9 (14): 2105-2108.

Wallace SM, Shah VP, Epstein WL, Greenberg J, Riegelman S, 1977. Topically applied antifungal agents. Percutaneous penetration and prophylactic activity against Trichophyton mentagrophytes infection. Archives of Dermatology, 113 (11): 1539-1542. 